\title{
Adaptive Educational Gameplay within Smart Multipurpose Interactive Learning Environment
}

\author{
Marko Divéky, Peter Jurnečka, Rudolf Kajan, Luboš Omelina, Mária Bieliková \\ Institution of Informatics and Software Engineering, Faculty of Informatics \\ and Information Technologies, Slovak University of Technology \\ Ilkovičova 3, 84216 Bratislava, Slovakia \\ bielik@fiit.stuba.sk
}

\begin{abstract}
Modern technologies have brought many innovations into the system of education. Learners now have an opportunity to educate with the help of electronic materials often provided by web-based infrastructures. Although the majority of learners - especially children or teenagers - find studying from such materials less exciting than playing today's popular computer games that, however, lack signs of education. In this paper, we describe an innovative concept of three-dimensional interactive educational games that combine the excitement and looks of popular computer games with the educational potential of electronic study materials. One of the key issues is adaptability (games are also playable by handicapped users) and adaptivity according to learners' level of knowledge. Together with automatic generation of educational games based on metadata provided by authors of educational materials, we enable the creation of educational games that have many shapes reflecting learners' needs.
\end{abstract}

\section{Introduction}

Education has significantly evolved for the last decade, mostly thanks to the Internet and its World Wide Web service, which enable learners to educate interactively and share their ideas and knowledge with each other - even if they are miles apart. Today, learners are presented with a countless number of diverse educational materials, such as multimedia encyclopedias or interactive online tutorials. Some of them are adaptive, and thus provide better support for individual learners [3].

Despite all these possibilities, many learners lack motivation into studying. Although there are some exceptions, the fact that we all need to be motivated more into studying yet endures. Educational games (i.e. serious games) are a new element that evolves rapidly [8]. Computer games offer something that learners cannot get from any present kind of electronic or printed educational materials - enjoyment and fun, both of which play a crucial role in effective learning [4]. Moreover, computer games do a great job in motivating $[2,6]$. However, the most popular ones are currently far away from being educational. Games intended for education not only often lack the thrill of popular computer games, but also do not allow teachers without programming expertise to alter their scenario. Furthermore, these games are, as a whole, not designed for handicapped players.

We propose an innovative concept that forms a unique solution for the above mentioned problems. It combines the advantages from both interactive educational materials and popular computer games by giving teachers (i.e. authors of educational materials) the ability to transform study materials into exciting educational games that can be played even by handicapped users (e.g., visually impaired or deaf). In this paper, we also present the S.M.I.L.E. system (Smart Multipurpose Interactive Learning Environment), which we have developed in order to prove our concept of automatic adaptive game generation.

\section{Educational Games Model}

Our model of educational games is based on today's most popular genre of computer games - Role Playing Games (RPGs) [7]. Such games are exciting, easy to control (what is especially important for handicapped users) and have a vast educational potential [4].

Games based on the RPG genre take place in a realistic world set in a specific time (e.g., in medieval age, at present time or in the future) or in an imaginary world that is or is not close to reality. Players are represented by and control an avatar (most commonly in a human form) and solve various quests throughout the game. These require the player to find certain 
objects which he needs to correctly use or combine in order to solve a particular problem, and/or require the player to choose a correct answer from a number of given answers to a certain question. Quests are assigned to players by Non-player Characters (NPCs) that cannot be controlled by players and interact with them through dialogs.

The proposed educational games model consists of two learning environments:

- Teacher-created games present a practical and enjoyable form of evaluating the players' level of knowledge and skills from a desired field. Games are specified by teachers either visually by defining their concept, or automatically generated by the system according to parameters set by the teacher. Although the first option already makes the described concept innovative, it is the latter option which makes it an interesting and unique one.

- A persistent virtual world that is made of quests based on all available educational materials, and therefore practically resembles one vast game that contains all possible educational quests. It is intended for self-training and raising the players' level of knowledge.

The only difference between the teacher-created games and the virtual world is that the games can also contain quests marked as private by their authors. Practically speaking, the described model encourages learners to first train and educate themselves by solving quests in the persistent virtual world, and afterwards to test their newly gained knowledge and skills in games that have been created by teachers to test the level of comprehension of a certain knowledge (from various subjects such as mathematics, chemistry or biology). Since these games can contain private quests (which are not available to players in the common virtual world), they represent a friendly way to check the level of learners' knowledge.

In case a learner has a problem completing a particular quest during gameplay, he has the possibility to view educational materials related to the problem that is tested by the quest (via the in-game Knowledge Viewer). Moreover, materials that may be helpful in completing the currently assigned quests are dynamically suggested to learners during gameplay.

The purpose of educational materials is to help players successfully complete the available educational games. All materials are collaboratively created, edited and managed by teachers and are stored in a format that makes it possible to adapt their formatting for each user's individual preferences.

\section{Automatic Generation of Educational Games}

The key to the automated generation of educational games is based on the fact that every stored educational material has a defined set of game objects associated to it and each game object can have one or more relationships defined with other objects (see Figure 1). For example, chemistry educational materials about water, sulfur trioxide and sulfuric acid (named "Water," "Sulfur Trioxide" and "Sulfuric Acid") may

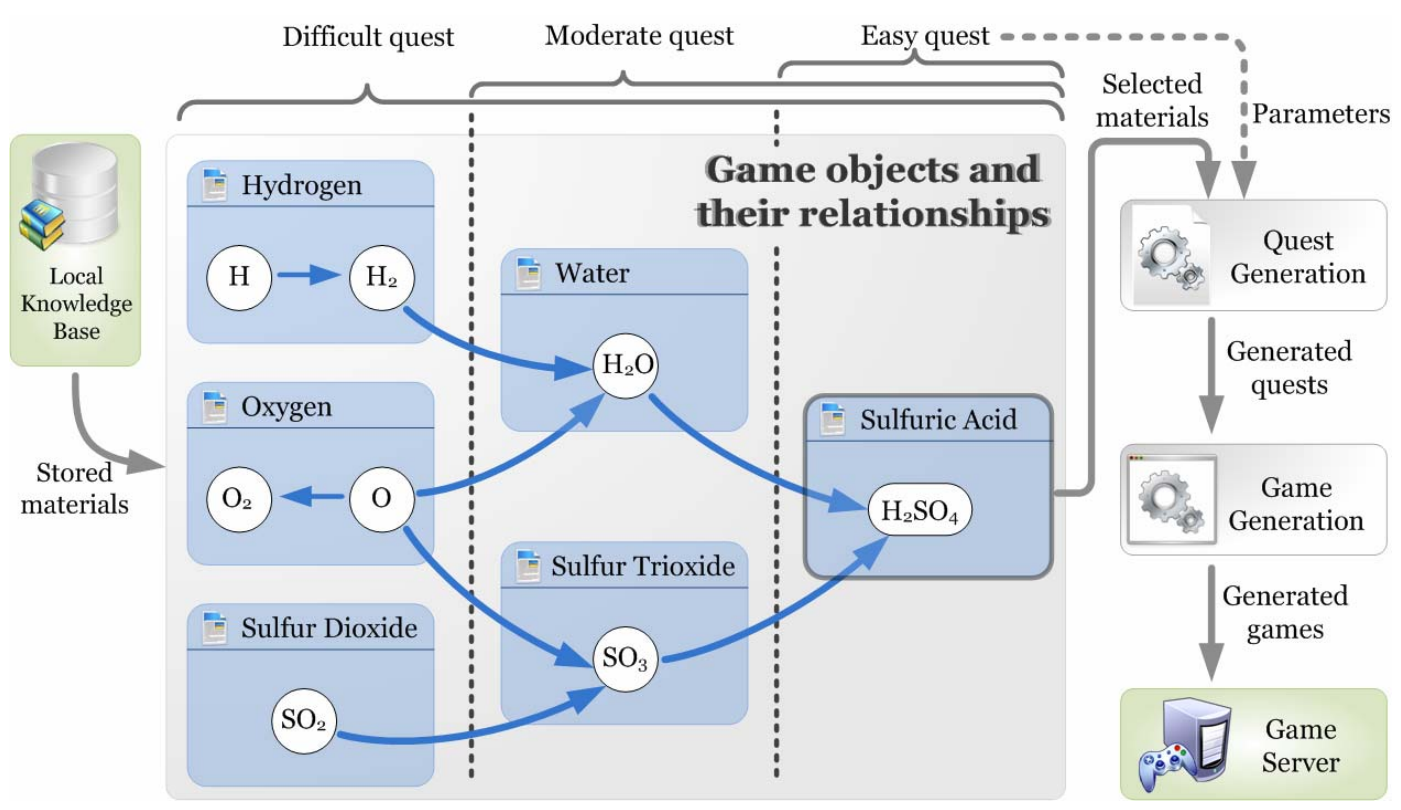

Figure 1. An example of automatic generation of educational games. 
have game objects such as " $\mathrm{H}_{2} \mathrm{O}$, " " $\mathrm{SO}_{3}$ " and " $\mathrm{H}_{2} \mathrm{SO}_{4}$ " associated to them respectively.

When a teacher chooses to generate a new quest or game based on an educational material (e.g., "Sulfuric Acid"), all game objects related to the chosen material are found (e.g., " $\mathrm{H}_{2} \mathrm{SO}_{4}$ "), their relationships with other game objects are examined (e.g., relationships with " $\mathrm{H}_{2} \mathrm{O}$ " and " $\mathrm{SO}_{3}$ ") and a set of interactions among them is created - forming a quest that learners accomplish throughout gameplay.

Based on parameters specified by the teacher - such as difficulty, the generated quests may require more or less effort and knowledge depth in order to be successfully completed. For example, if the difficulty was set to easy, the resulting quest will require the players to only find " $\mathrm{H}_{2} \mathrm{SO}_{4}$ " throughout the game. In case the difficulty was set to moderate, students will have to find both " $\mathrm{H}_{2} \mathrm{O}$ " and " $\mathrm{SO}_{3}$ " and afterwards mix them together correctly in order to complete the generated quest. A quest of high difficulty would not only require the players to find all objects in any way related to " $\mathrm{H}_{2} \mathrm{SO}_{4}$," but also to combine them correctly.

In the process of game generation, the inputted quests are analyzed and the resulting game's landscape is generated according to properties of game objects (such as their place of occurrence - e.g., a desert or forest) contained in the quests. Afterwards, the quests are placed throughout the whole generated landscape.

Automatic educational game generation consists of two separate phases: the Quest Generation and Game Generation phases that utilize artificial intelligencebased algorithms for solving particular tasks (e.g., simulated annealing to find appropriate places during the landscape generation process or depth search strategy with backtracking for quest generation).

\section{Quest Generation:}

1. Analysis of game objects related to specified knowledge, including their relationships.

2. Formation of a partially ordered set of in-game interactions from the analyzed relationships.

3. Creation of generic NPCs who assign and explain the generated quests to players and reward those who have successfully accomplished them.

Game Generation:

1. Content analysis of all specified quests, together with game objects that they contain.

2. Landscape generation based on properties of the analyzed game objects.

3. Grouping of generic NPCs (from quests) into specific in-game NPCs, since a single in-game NPC can be responsible for and handle the assignment, explanation and accomplishment of multiple quests.
4. Generation of textual dialogs for the created ingame NPCs.

5. Placement of all quests and in-game NPCs onto the generated landscape.

6. Generation and positioning of waypoints for the movement of severely sight-handicapped and blind players throughout the landscape.

\section{Gameplay Adaptability and Adaptivity}

The proposed concept of educational games was designed with both adaptability and adaptivity in mind. Speaking about adaptivity, we point out that the system attempts to be different for different learners and groups of learners by taking into account information accumulated in learner's or group's profiles. Speaking about adaptability, we point out that the system can be adapted by every user according to his needs and abilities (mostly related to possible handicap of users).

\subsection{Adaptability of Educational Materials}

Educational materials contain anything ranging from formatted text and lists to pictures, videos, audio files and other multimedia materials, such as Macromedia Flash elements. Each material is represented in an XML format designed especially for this purpose. As a consequence to the fact that the format separates the materials' content from their formatting, we are able to let each user specify how he would like to format all viewed educational materials (e.g., learners with a vision impairment can set the size of all displayed text and have all multimedia elements shown larger, deaf learners can set the option to display available subtitles below every video or audio file).

The mechanism of formatting educational materials according to users' preferences is shown in Figure 2. Each user has a profile containing his preferences according to which all viewed materials are formatted - differently for each and every user. These preferences contain various formatting parameters (e.g., the font face, size and color used for text rendering, the zoom factor of all viewed multimedia elements and the background color of documents). Users are able to choose from a set of predefined formatting presets based on standardized schemes (e.g., see a preset designed for color-blind learners, right part of Figure 3) or may further customize these presets.

The specified settings serve as parameters for the corresponding XSL transformations - the transformation into HTML and the transformation into the format used by the in-game Knowledge Viewer. The XSL transformations take the viewed knowledge as XML input and the user's settings as their parameters, and then transform the knowledge into formats used by 


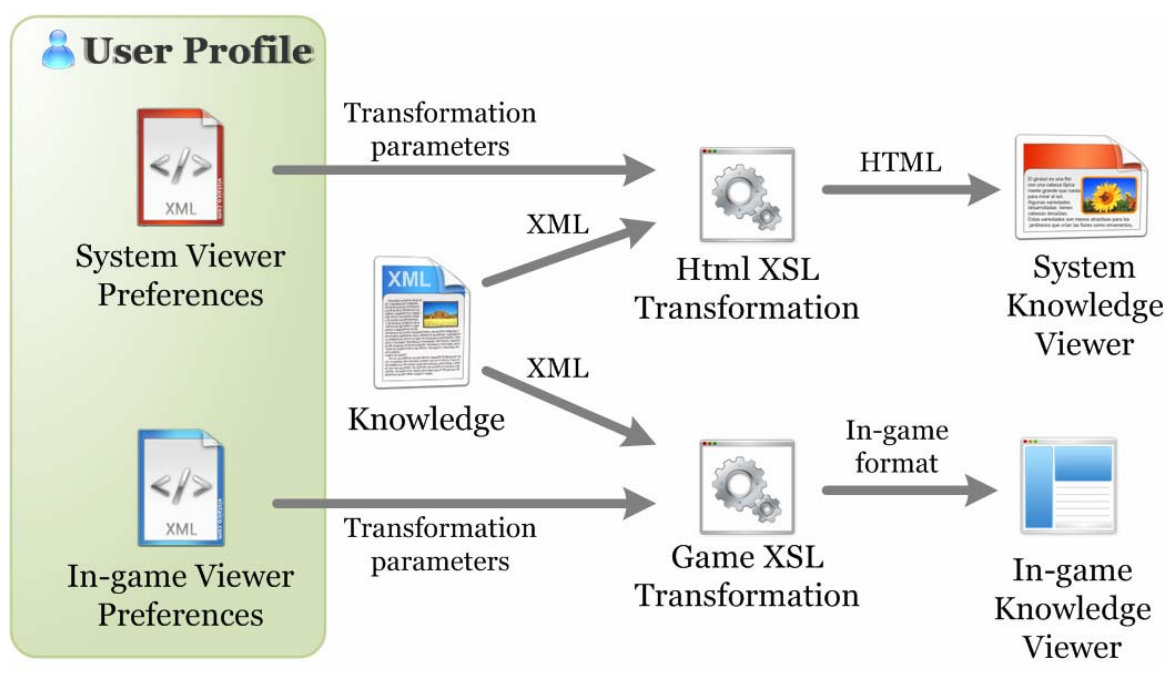

Figure 2. Formatting of educational materials.

the viewers. The resulting documents are formatted according to users' individual preferences.

Thanks to the fact that both educational materials and the knowledge viewers' formatting settings are stored in XML format, it is possible to replace them (along with both XSL transformations) with a completely different XML representation, mainly for the integration with other existing bases of educational materials, including e-learning standards [5].

\subsection{Adaptability of User Interfaces}

To provide interfaces for all, we designed the educational games with the possibility to be fully controlled and interacted with via voice recognition and also with peripherals for the handicapped, such as Braille keyboards. Handicapped learners may also use the keypad of their mobile phone or PDA as an input device. All these interfaces enable handicapped learners to fully control and interact with the games.

For example, learners with a sight handicap control all interfaces with voice commands and have the contents of all educational materials read to them using a computer-synthesized voice. Moreover, such learners have all text in the educational materials shown as large as they prefer. Similarly, deaf players are presented with subtitles below every video or audio file.

Games can be played by means of voice-activated commands using a version of the Game Client specialized for blind and vision-impaired users. It reads all available in-game actions to users via computer voice synthesis and lets them speak which actions to take back at the system. To other players, a blind player looks just like any other player, with the exception that he moves according to waypoints automatically placed when the game is created. Players with minor vision impairment can customize the visual appearance of important in-game elements, such as NPCs - as shown in Figure 4. Deaf players are informed of every in-game event visually, not audibly.

All in all, specialized interfaces enable handicapped learners to participate in the educational process by

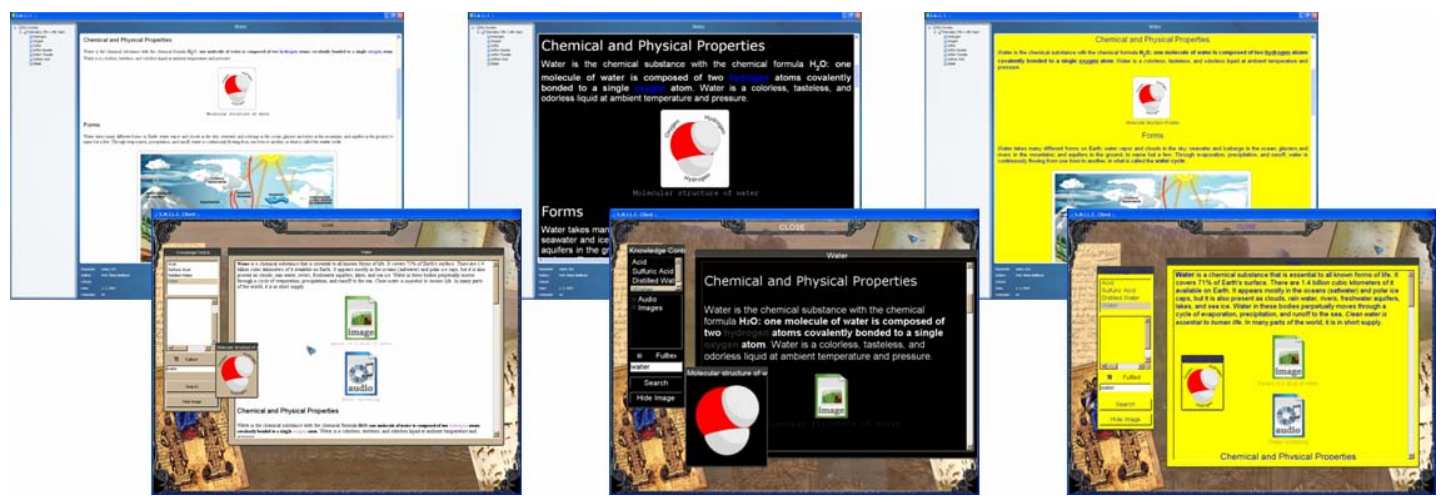

Figure 3. Adaptability of user interfaces for various disabilities. 


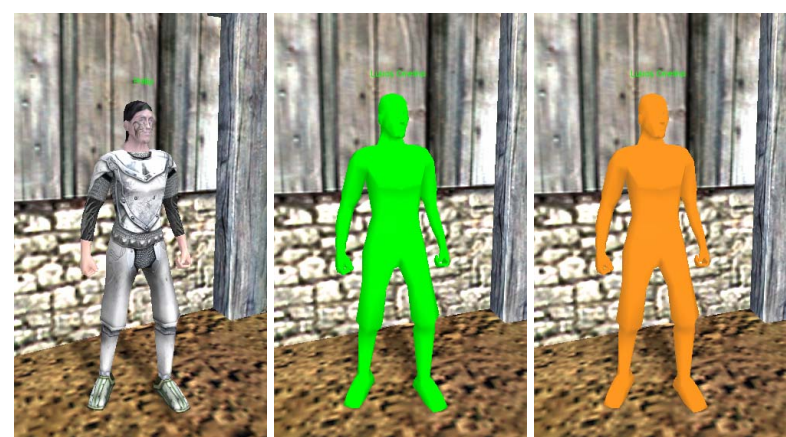

Figure 4. Adapting NPC for visually impaired.

playing educational games with their non-disabled friends, and thus socialize with the community.

\subsection{Adaptivity of Game Content}

Every quest, whether it was manually designed by a teacher or automatically created according to his preferences, is based on a set of educational materials since each game object covered in the quest is associated to a particular knowledge material. In addition, each educational material is tied to a specific subject or field (such as mathematics). Having all this in mind, it is possible to classify all quests according to subjects that they are related to and estimate how good each player is at each subject based on how he managed to solve particular quests.

For example, if a quest requires a student to find game objects " $\mathrm{H}_{2} \mathrm{O}$ " and " $\mathrm{SO}_{3}$," to combine them into " $\mathrm{H}_{2} \mathrm{SO}_{4}$ " and to answer a question about the physical properties of water, then the quest is related to chemistry and physics - presuming that " $\mathrm{H}_{2} \mathrm{O}$, " " $\mathrm{SO}_{3}$ " and " $\mathrm{H}_{2} \mathrm{SO}_{4}$ " are associated to educational materials all tied to chemistry, and the answered question associated to a physics material. If the student successfully solves this quest, we increase the estimated level of his skills in chemistry and physics. Similarly, if a player is unable to solve the specified quest, his estimated skills will decrease based on what he was unable to accomplish: if he incorrectly combined " $\mathrm{H}_{2} \mathrm{O}$ " with " $\mathrm{SO}_{3}$," his estimated chemistry level would decrease. In case he incorrectly answered the specified quest's question, it would be the physics level decreasing.

Thanks to the fact that we estimate the level of knowledge of each subject for every player and the fact that each quest is classified according to subjects it is related to, all educational games are adapted individually to every player's (estimated) level of knowledge and skills. Players are dynamically navigated into solving quests (e.g., by pointing players at map locations of the in-game NPCs that assign them) most suitable for their estimated knowledge level. Additionally, while solving quests, options of the appropriate difficulty are chosen for players to solve (e.g., if a player has his estimated knowledge of physics at a low level, he will need to answer a simpler physics question in order to solve the above described quest - and vice-versa).

By utilizing the Item Response Theory [1], we are able to predict how will players react to both tasks and questions they are given while solving quests, and thus to measure their level of forgetfulness by testing them on tasks and questions they have previously successfully completed and answered.

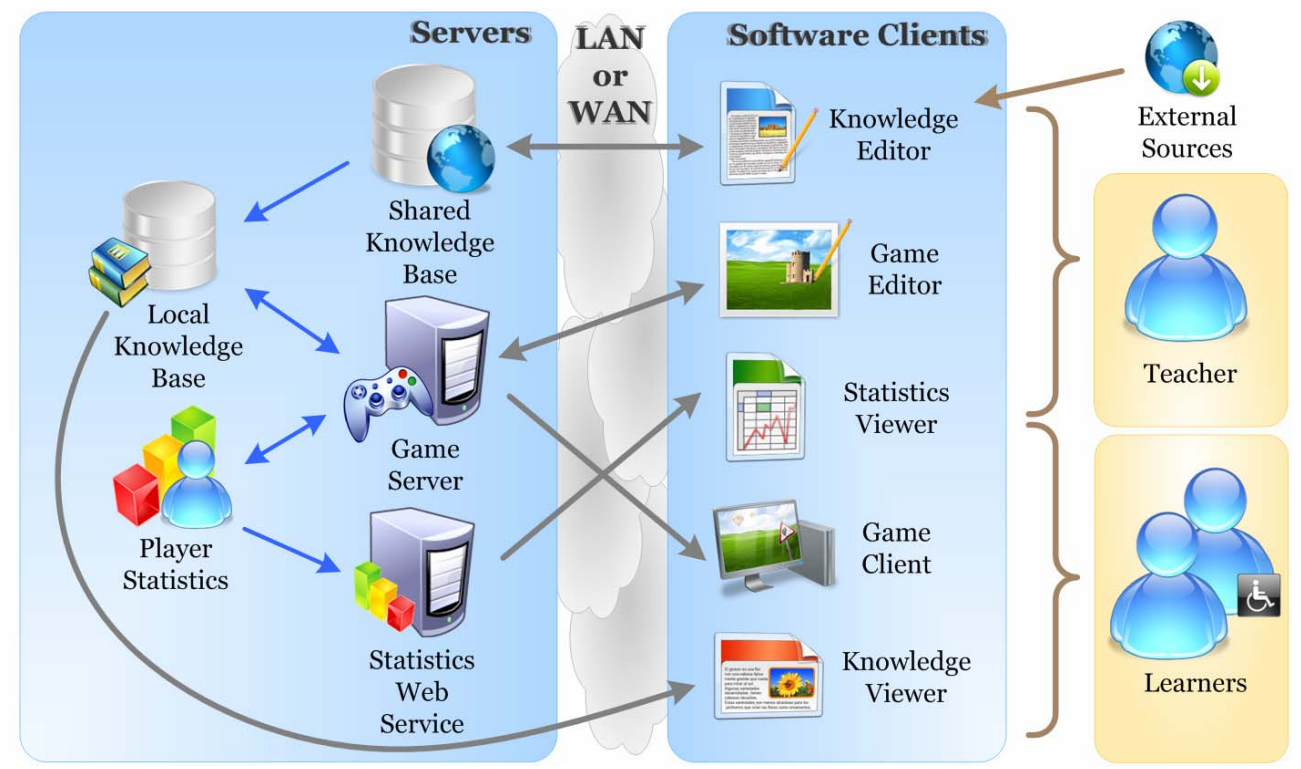

Figure 5. Overview of the S.M.I.L.E. system. 


\section{Concept Validation}

We have experimented with the proposed concept of automatically generated adaptive games by developing the S.M.I.L.E. system (Smart Multipurpose Interactive Learning Environment). An overview of the system is depicted in Figure 5.

Knowledge Editing. The creation or import of educational materials is done with the assistance of the Knowledge Editor, which is a full-featured WYSIWYG editor designed to ease the process of creating multimedia materials for educational games created by the S.M.I.L.E. system. One of the most assistive functions of the editor is the feature to automatically search the Internet for multimedia elements (pictures and audio files) related to the topic of the currently edited knowledge. Chosen elements can be easily inserted (respecting copyright issues) into the current material by drag 'n' drop.

Game Editing. Teachers can collaboratively edit, share and use the stored materials for teaching their pupils. Most importantly, teachers are presented with the ability to create their own educational games based on these knowledge materials via the Game Editor.

Data Storage. All created games including the necessary maps, textures, models and sounds are stored in the Game Server, whereas all study materials, quests and game objects along with their relationships are stored in the Local Knowledge Base, to where study materials are copied from the Shared Knowledge Base by the teachers' version of the Knowledge Editor. This mechanism not only reduces requests off the Shared Knowledge Base - which can become a bottleneck of the whole system, but also ensures that the necessary study materials are accessible even when connectivity to the Shared Knowledge Base gets interrupted.

Game Types. Learners have the option to educate by playing both games created by their teachers (which are based on quests either directly chosen by teachers, or on quests adaptively selected according to parameters set by the teachers), and also by playing in a virtual world consisting of all quests that are stored in the system and marked public by their authors.

Knowledge Viewing. To ease the process of learning, learners can browse and view all knowledge materials available in the Local Knowledge Base via the Knowledge Viewer, which formats the content of displayed materials according to every learner's preference. It also allows learners to search for available study materials via keywords or by browsing through study materials that are tied to courses they attend. The viewer is also accessible in-game, so learners can view the related study materials without interrupting the gameplay.

\section{Conclusions}

The main contribution of the presented work is in devising a novel concept of three-dimensional adaptive educational games that can be automatically generated. A unique feature is the proposal of methods that allow teachers themselves to define educational games based on the stored educational materials without requiring any knowledge of programming. Three-dimensional educational games (including the persistent virtual world) are dynamically generated based on teachers' preferences. User interfaces are designed as adaptable in such a way that they support handicapped learners.

In order to validate the presented concept, we have designed and implemented a system called S.M.I.L.E. that encapsulates and combines the advantages of two distinct worlds - interactive educational materials and popular computer games. It also enables handicapped learners to educate together with their non-disabled colleagues. Such concept has not yet been realized to our knowledge in any of the existing applications.

We have had positive feedback on the adaptability and adaptivity features from both teachers and students at elementary and secondary schools for handicapped children, where we demonstrated our system.

\section{Acknowledgements}

This work was partially supported by the Cultural and Educational Grant Agency of the Slovak Republic, grant KEGA 3/5187/07, and was developed within the Software Design category of Imagine Cup 2007.

\section{References}

[1] Baker F., The Basics of Item Response Theory, ERIC Clearinghouse on Assessment and Evaluation, University of Maryland, College Park, MD, 2001.

[2] BBC News, Computer games 'motivate pupils', 2006, [online] news.bbc.co.uk/1/hi/education/4608942.stm

[3] Brusilovsky, P., Peylo, C., "Adaptive and intelligent Web-based educational systems", Int. Journal of Artificial Int. in Education 13 (2-4), 2003, pp. 59-172.

[4] Dryden, G., Vos, J., The Learning Revolution, Jalmar Press, Austin, 1999.

[5] Fallon, C., Brown, S., E-Learning Standards: A Guide to Purchasing, Developing, and Deploying StandardsConformant E-Learning, CRC Press, 2002.

[6] Feller, B., Video games can reshape education, Associated Press, 2006, [online] http://www.msnbc.msn.com/id/15309615

[7] Roleplay.org, Role-playing Game Introduction, [online] http://www.roleplay.org/articles/rpg-intro/

[8] Zyda, M., Creating a Science of Games, Communications of the ACM, 50 (7), 2007, pp. 27-29. 